\title{
The First 10 Years of NeuroIS: A Systematic Literature Review of NeuroIS Publications (2007 - 2017)
}

\author{
Md Rasel Al Mamun \\ University of North Texas \\ mdrasel.mamun@unt.edu
}

\author{
Dan J. Kim \\ University of North Texas \\ Dan.Kim@unt.edu
}

\author{
Alsius David \\ University of North Texas \\ alsius.david@unt.edu
}

\author{
Bin Mai \\ University of North Texas \\ Bin.Mai@unt.edu
}

\author{
Thomas D Parsons \\ University of North Texas \\ Thomas.Parsons@unt.edu
}

\begin{abstract}
NeuroIS is an emerging and promising academic field that has attracted increasing attention. The year 2017 signifies the $10^{\text {th }}$ year of existence of NeuroIS as a research field in information systems area. In this study, we conduct a systematic literature review of the NeuroIS academic research publications of last 10 years (2007-2017). As a result, we categorize the existent NeuroIS literature into 8 groups, explore the correlations among various NeuroIS concepts/ constructs, and demonstrate how the study enhances our understanding of the granulated interrelationships between pairs of NeuroIS elements. The implications of the result to the NeuroIS research community are discussed.
\end{abstract}

\section{Introduction}

NeuroIS ${ }^{1}$ has emerged over the past decade as a significant new IS research field that has attracted increasing attention. More and more researchers have started investigating what neuroscience can do about the human interactions with information systems. To engage in this developing endeavor, it is thus important to have a synthesis of the research publications so far in the field of NeuroIS, identify the research streams existing in current literature, and explore the relationships among the elements important to NeuroIS research. Such an effort would better inform researchers about the state-of-the-art of NeuroIS, and facilitate their efforts in making further contributions to the field of NeuroIS. This synthesis what our paper is focusing on.

The emergence of NeuroIS was preceded by the phenomenon that neuroscience has been extensively studied as a key to understanding human behaviors [1,

1 The term NeurolS (Neuro-Information-Systems) has been addressed by Dimoka et al. [2007] to describe the idea of applying cognitive neuroscience theories, methods, and tools in
2]. This approach has been applied in a wide variety of fields such as social science [3-6], economic science and computer science [7-9]. Since the Information Systems (IS) field tries to explain human behavior in the interactions with information technology, in accordance with the trend of advance of neuroscience in other fields, IS researchers have also begun to investigate the potential of neuroscience for IS research. With the increased use of Neuroscience methods and tools in scientific fields closely related to IS, scholars have introduced the concept of NeuroIS into the IS literature $[10,11]$. The concept of NeuroIS emerged in the course of the 2007 International Conference on Information Systems[10]. Now NeuroIS research is defined as "applying cognitive neuroscience theories, methods, and tools to inform IS [information systems research" [12]. More specifically, Riedl, Banker [13] suggested that NeuroIS "relies on neuroscience and neurophysiological theories and tools to better understand the development, use, and impact of information technologies (IT). NeuroIS seeks to contribute to ... the development of new theories that make possible accurate predictions of IT-related behaviors, and ... the design of IT artifacts that positively affect economic and non-economic variables (e.g., productivity, satisfaction, adoption, well-being)”.

Using biological signals generated from the human body during humans' interactions with IS, researchers can directly measure the constructs that underlie human behavior, particularly affective and subconscious effects and significant new insights had been obtained that shed lights on various IS phenomena. Some representative examples of NeuroIS research include [14-21].

Although the NeuroIS field is still in a nascent stage, it is suggested that IS scholars become more familiar with the methods, tools, and measurements, research domains, theory types that have already been

Information Systems (IS) research. Although NeurolS covers other domains such as articifical intelligence, neural networks, etc., this study mainly focuses on the publications in IS domain. 
associated with NeuroIS field [13]. A better understanding of these NeuroIS-relevant elements and their inter-relationships will help IS researcher to obtain sound methodological knowledge about the landscape of the current NeuroIS field, to be better informed about how to engage in NeuroIS research, and to leverage the full potential of Neuroscience for IS research [22].

So far, an increasing number of scholars have applied various methodologies, adopted various tools, and analyzed various types of measurements when investigating IS research questions [23-30]. These contributions and discussions emerging in the NeuroIS literature have contributed to an increased understanding of, and interest into, NeuroIS.

However, we notice that there are only limited number of research efforts that survey/synthesizes the current NeuroIS research, describing in a systematic and comprehensive manner the correlation among tools, contribution to IS, used theory, research domain, reference discipline and measurement of constructs that is essential to an increased understanding of NeuroIS research. Among the existing literature, a study [31] provided a solid theoretic framework that illustrates what elements should be relevant in NeuroIS research. The study [31] is prescriptive in nature, suggesting what should be done in NeuroIS research.

The purpose of this study is to conduct a systematic literature review (SLR) on the first ten years (i.e., from the inception of NeuroIS in 2007 to 2017) of NeuroIS academic publications to provide a descriptive overview for the first decade of NeuroIS. That is, we want to describe what had actually been done in NeuroIS during the past decade. Based on the theoretic framework provided by [31], we investigate how the existing NeuroIS literature establish the interrelations among different NeuroIS reference disciplines, what types of contributions they made to the IS understanding, what types of neurophysiological tools had been used, what conceptual constructs had been measured, what research domains had been addressed and what theory types had been adopted, and therefore fill the void of a much needed state-of-the-art report on the current landscape of NeuroIS research. More specifically, we attempt to address the following research questions: how many categories can the current NeuroIS research be classified into, and what are the properties of those categories? How are the tools, research domains, theory types and category of constructs utilized in the NeuroIS publications associated with the various types of contributions those publications aim to make? How are the various components of NeuroIS research publications (e.g., tools, domains, constructs, etc.) inter-related?
The reminder of this paper is organized as follows. In section 2, we describe the methodology used in this study and the data extraction process used. In section 3, we present the data analysis process and results. In section 4 , discusses conclusion, limitation and future research direction.

\section{Research Methodology}

In this study, the PRISMA [30] guidelines has been implemented to conduct a systematic literature review. The PRISMA guidelines were developed to provide a standard framework to guide the process of systematic literature review and meta-analysis. Since its inception in 2009, it has been adopted by researchers in a wide range of fields.

\subsection{Literature search strategy and selection criteria}

For the purpose of this study, we include research articles from different disciplines such as neuropsychology and NeuroIS those had utilized neuroscience tools (e.g., fMRI, EEG, eye-tracking devices, etc.) to study specific IS phenomena (i.e., we do not include those positioning papers such as [23, 24, 32]). First of all, we search through Google Scholar as well as several proprietary academic research databases using the key term of NeuroIS. Then we focus on the NeuroIS literature that does not directly bear the term "NeuroIS". This research literature we selected to be included in our SLR analysis are from three main sources: 1 ) the NeuroIS research repository available in NeuroIS.org keeps track of the NeuroIS related publications over the years, and includes all Gmunden Retreat on NeuroIS, the leading academic conference dedicated to NeuroIS research, publications since the conference's inauguration in 2009. We started collecting all the NeuroIS publications listed in NeuroIS.org; 2) we then searched the IS journals and academic research databases using key terms including neuroscience, brain function, neurophysiology, psychophysiology and other similar terms. From the result, again, we included only those that had utilized NeuroIS tools to investigate IS phenomena. 3) We then searched the neuroscience/psychology academic research databases (PsycINFO, PubMed) using terms including information systems, information technology, cyberspace, digital environment, Internet and other similar terms. After excluding those that focus on the medical analysis and treatment as the main research question, a total of 131 published journal and conference articles were collected for the analysis of the study. Appendix 1 lists those collected articles. 


\subsection{Analysis: coding and data extraction}

For the coding of collected articles from the NeuroIS literature, we adopt the NeuroIS framework as described in [31], in which the following 6 elements are identified as integral to NeuroIS research:

- Reference disciplines of NeuroIS: the scientific disciplines that provide insights into biological foundation and application relevant to human interaction with IS. Riedl and Léger [31] identified 10 relevant scientific disciplines that significantly inform the development of NeuroIS. Those disciplines can be categorized into fundamental research, which is more of theoryoriented, and applied research, which is more of design-oriented.

- Contributions to the NeuroIS research: applying neurobiological approaches to IS research and practice can result in significant new insights into IS phenomena. Riedl and Léger [31] identified 10 potential contributions of neuroscience to IS research. Similarly, those contributions can be categorized into either a theoretic focus or a design focus.

- Tools used in NeuroIS research: a wide variety of neuro-physiological tools are available to facilitate IS research. Corresponding to the human neural systems, these neuro-physiological tools can be categorized into three kinds based on their measuring type (i.e., central nervous systems (CNS), peripheral nervous system (PNS), and hormone systems).

- Neuroscience theories used to inform NeuroIS research: Riedl and Léger [31] proposed a taxonomy of neuroscience theories from a NeuroIS perspective, and categorized the relevant theories into three types (i.e., analysis, explanation, and design and action).

- Constructs of interests to NeuroIS research: for the different constructs that are relevant to NeuroIS research, Dimoka, Pavlou [33] developed a list of 34 such constructs, and grouped them into 4 categories (i.e., cognitive processes, emotional processes, social processes, and decision-making processes).

- Research domains for NeuroIS: NeuroIS research can include a wide variety of topics, and Dimoka, Banker [32] grouped them into 3 research domains (i.e., development and use of systems, IS strategy and business outcomes, and group work and decision support).
Following the NeuroIS framework, we coded the collected NeuroIS literature. First, a standard binary coding protocol was developed for abstracting the six elements from the articles. For each selected paper, we record the citation information and review the content of the article to check to which categories of the six elements that the article related to. For each category of each element, a coder assigned " 1 " if the particle covers to the category, or " 0 " otherwise. The coder had repeated this step for all identified articles.

\section{Data analysis and results}

Descriptive statistics and text mining techniques (e.g., Text-parsing, Text-filter and Text-cluster Nodes in SAS Enterprise Miner) have been applied to analyze the data.

\subsection{Descriptive statistics: distribution of NeuroIS articles}

Through descriptive statistics, we observed the following patterns of the NeuroIS research so far:

- The most referenced disciplines in NeuroIS research are neuropsychology, cognitive neuroscience, and decision neuroscience. The least referenced disciplines are neuro-ergonomics and affective computing.

- About $47 \%$ of the NeuroIS research articles we analyzed are of theoretic focus and 53\% are of design focus.

- About $51 \%$ of the NeuroIS research utilize neuroscience tools measuring CNS (with fMRI and EEG as two most common tools), $44 \%$ utilize tools measuring PNS (with eye-tracking devices and EDA skin sensor as two most common tools). $5 \%$ of utilize tools measuring hormone systems.

- $45 \%$ of NeuroIS research we analyzed investigate cognitive process constructs, $24 \%$ investigate decision-making process constructs, $20 \%$ investigate emotional process constructs, and $10 \%$ investigates social process constructs.

- In terms of research domain, $60 \%$ of NeuroIS research articles we analyzed are about the development and use of systems, $22 \%$ are about group work and decision support, and $18 \%$ are about IS strategy and business outcome.

- $\quad 42 \%$ of the NeuroIS research articles we analyzed are informed by the "explanation" type of neuroscience theories, and the rest is split evenly between the "analysis" type and "design and action" type. 


\subsection{Text cluster analysis: categorization of NeuroIS articles}

We are interested in investigating the interrelationships among this seemingly disparate collection of literature. It is reasonable to assume that some of the articles share certain common properties with some other articles and thus it can be said that they belong to the same type of NeuroIS research. In order to explore this issue, we perform a text cluster analysis.

Text mining using text clustering involves the sorting of documents into distinct groups based on shared descriptive terms. The process uses singular value decomposition (SVD), Log-Entropy weighting and an Expectation maximization algorithm and is performed using the Text-Parsing Text-Filter and Text-Cluster Nodes in SAS Enterprise Miner.

To determine the number of clusters for our analysis, we follow Can and Ozkarahan [34] and define the collection of NeuroIS literature data as a matrix of size 131 by 50 , where 131 is the number of document, and 50 is the number of terms we used for coding (i.e., 10 terms for reference discipline, 10 for contribution, 20 for neuroscience tool, 3 for neuroscience theory type, 4 for construct type, and 3 for research domain). The number of all the non-zero entries in the collected dataset is 837. Therefore, based on Can and Ozkarahan [34], the number of clusters can be calculated as $131 * 50 / 837=7.82$. Therefore, we specify 8 clusters. This number is confirmed by the SAS Text-Cluster Nodes, which also generate 8 clusters when we do not specify the number of clusters in the configuration. The resultant 8 clusters can be interpreted as 8 types of NeuroIS research in the current literature. The following table reports the 8 clusters and their properties.

Table 1. The 8 Clusters of NeuroIS Research

\begin{tabular}{|c|c|c|c|c|c|c|}
\hline Cluster & Focus & Discipline & Tools & Theory & Constructs & $\begin{array}{c}\text { Research } \\
\text { domain }\end{array}$ \\
\hline 1 & Design & Fundamental & PNS & $\begin{array}{c}\text { design \& } \\
\text { action }\end{array}$ & cognitive & $\begin{array}{c}\text { Development } \\
\text { and use of } \\
\text { system }\end{array}$ \\
\hline 2 & Design & Fundamental & PNS & explanation & emotional & $\begin{array}{c}\text { IS strategy and } \\
\text { business } \\
\text { outcomes }\end{array}$ \\
\hline 3 & Design & Applied & CNS & analysis & $\begin{array}{c}\text { Decision } \\
\text { making }\end{array}$ & $\begin{array}{c}\text { Development } \\
\text { and use of } \\
\text { system }\end{array}$ \\
\hline 5 & Theory & Fundamental & CNS & explanation & cognitive & $\begin{array}{c}\text { Group work and } \\
\text { decision support }\end{array}$ \\
\hline 6 & Theory & Fundamental & PNS & Analysis & Social & $\begin{array}{c}\text { IS strategy and } \\
\text { business } \\
\text { outcomes }\end{array}$ \\
\hline 7 & Theory & Fundamental & PNS & analysis & $\begin{array}{c}\text { Decision } \\
\text { making }\end{array}$ & $\begin{array}{c}\text { IS strategy and } \\
\text { business } \\
\text { outcomes }\end{array}$ \\
\hline
\end{tabular}

\begin{tabular}{|c|c|c|c|c|c|c|}
\hline 8 & Theory & Applied & PNS & analysis & Cognitive & $\begin{array}{c}\text { IS strategy and } \\
\text { business } \\
\text { outcomes }\end{array}$ \\
\hline
\end{tabular}

From Table 1, it can be observed that there are 8 types of NeuroIS research. The first type (cluster 1) utilizes design and action type of theories from other fundamental research disciplines to study cognitive process constructs, using primarily neuroscience tools measuring PNS, we can call this type of NeuroIS research as cognitive research in IS design and development. The second type of NeuroIS research (cluster 2) attempts to utilize PNS tools to explain the constructs of emotional processes in order to inform the development of IS strategy and impact the resultant business outcome. We can call this type as emotional process impacts on IS business strategy. The third type (cluster 3) analyzes the CNS correlates associated with decision making process constructs in order to inform the design and development of IS. We can call this type as IS design and development decision making. The fourth type (cluster 4) attempts to explain the CNS activities associated with social process constructs to improve group interaction and decision support. We can call this type as social process factors to help group decision support. The fifth type (cluster 5) also studies the group decision support, but from a more theoretic perspective, focusing more on the cognitive process constructs that impact group decision support. We can call this type as the cognitive process factors to understand group decision support. The sixth type (cluster 6) analyzes the social process constructs and their relationships with IS strategy and business outcome. We can call this type as social process constructs and their impacts on IS strategy and outcome. The seventh type (cluster 7) utilizes PNS activities to analyze decision making process constructs to understand IS and business outcome. We can call this decision making factors and their relationships with IS strategy and business. And last but not least, the eighth type (cluster 8) attempts to analyze cognitive process constructs related to IS strategy and business outcome in order to improve the business outcome of IS strategy. We can call this as cognitive factors to facilitate IS strategy and improve business outcome.

These results can facilitate researchers engaging in NeuroIS research in several ways. First of all, they can see how their NeuroIS research efforts fit into the big picture of NeuroIS by identifying which type of NeuroIS research they are conducting. Also, NeuroIS researchers can also identify potential new research opportunities from Table 1 by incorporating elements absent from a cluster into that specific cluster. For example, can we attempt to utilize CNS neuroscience tools to investigate emotional constructs that are significant in various specific research domains? 


\subsection{Association analysis: linking of NeuroIS concepts}

In this section, we present some results from our attempts to explore the inter-relationships among the various concepts in NeuroIS. As described above, we review the existing NeuroIS literatures to identify various NeuroIS concepts. It is reasonable to assume that those concepts are inter-related. We thus conduct an association (market basket) analysis [35] and generate concept linking diagrams to illustrate the correlations among different NeuroIS concepts.

Association (market basket) analysis identifies association rules between items which occur frequently together, for example, keywords which occur frequently together among a set of articles or grocery items bought frequently together among a set of transactions [35]. This analysis also produces the confidence, support and lift values for each identified association rule. Concept link diagrams illustrate the strengths of various terms associated with a selected term. This selected term is the term that all the lines are connected to. It is located in the center of the diagram. Those terms most strongly associated with the selected term will show up in this diagram.

In this paper, due to the length limitation, we present only two results of concept association as examples. Figure 1 shows the concept linking diagram for the research concept of "design and action" as a neuroscience theory type. In Figure 1, it is noticed that NeuroIS research utilizes the "design and action" type of neuroscience theory, these theories usually contribute to the IS research by informing the design of IT artifacts, the research often references the disciplines of brain-computer interaction and cognitive neuroscience, and it frequently utilizes neuroscience tools including EEG and eye tracking devices. Table 2 shows the support, confidence, and lift of some of the association rules identified.

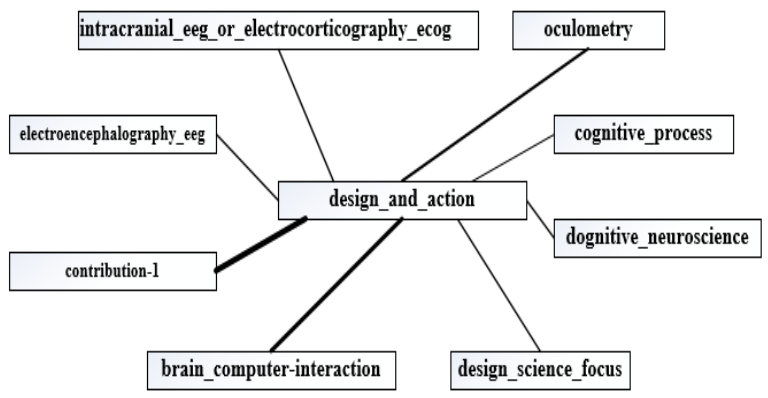

Figure 1. Concept linking diagram for the concept "Design and Action"

Table 2. Some association rules for the concept "Design and Action"

\begin{tabular}{|c|c|c|l|}
19.85 & 65.00 & 1.37 & Design_and_action ==> Cognitive_processes \\
\hline 19.85 & 65.00 & 1.09 & $\begin{array}{l}\text { Design_and_action ==> } \\
\text { Development_and_use_of_system }\end{array}$ \\
\hline 16.79 & 55.00 & 2.06 & Design_and_action ==> Contribution-1 \\
\hline 15.27 & 50.00 & 1.52 & Design_and_action ==> Cognitive_Neuroscience \\
\hline 12.98 & 42.50 & 1.47 & Design_and_action ==> Oculometry \\
\hline 11.45 & 37.50 & 1.26 & $\begin{array}{l}\text { Design_and_action ==> } \\
\text { Electroencephalography_EEG }\end{array}$ \\
\hline 6.11 & 20.00 & 1.05 & $\begin{array}{l}\text { Design_and_action }==> \\
\text { IS_strategy_and_business_outcomes }\end{array}$ \\
\hline 3.82 & 12.50 & 2.73 & Design_and_action ==> Brain-computer-interaction \\
\hline 2.29 & 7.50 & 1.23 & Design_and_action ==> Decision_Neuroscience \\
\hline
\end{tabular}

Note: S: Support, C: Confidence

Similarly, Figure 2 shows the concept linking diagram for the research concept of "IS strategy and business outcomes" as a research domain. From Figure 2, it can be observed that for NeuroIS research in the "IS strategy and business outcomes" domain, the neuroscience theories often contribute to the IS research by providing real-time bio-feedbacks on a user's biological state. The research often references the disciplines including consumer neuroscience and social neuroscience, and the research often involves the measurement of hormone systems. Table 3 shows the support, confidence, and lift of some of the association rules identified

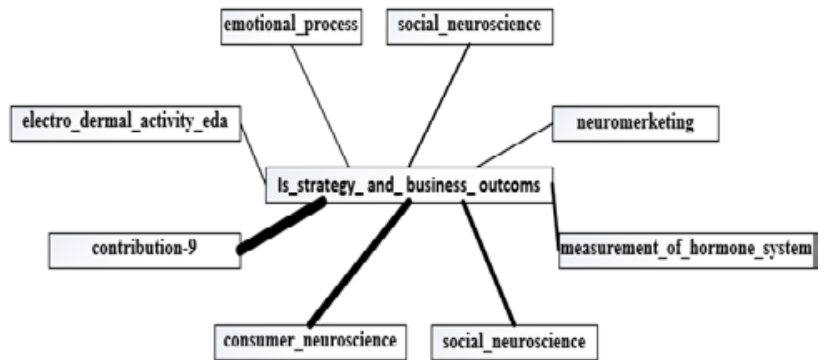

Figure 2. Concept linking diagram for the concept "IS Strategy and Business Outcomes"

Table 3. Some association rules for the concept "IS Strategy and Business Outcomes"

\begin{tabular}{|c|c|c|l|}
\hline S & C & Lift & Rule \\
\hline 10.69 & 56.00 & 1.15 & $\begin{array}{l}\text { IS_strategy_and_business_outcomes ==> } \\
\text { Theoretical_focus }\end{array}$ \\
\hline 9.16 & 48.00 & 1.01 & $\begin{array}{l}\text { IS_strategy_and_business_outcomes ==> } \\
\text { Cognitive_processes }\end{array}$ \\
\hline 8.40 & 44.00 & 1.07 & IS_strategy_and_business_outcomes ==> Explanation \\
6.11 & 32.00 & 1.07 & $\begin{array}{l}\text { IS_strategy_and_business_outcomes ==> } \\
\text { Electroencephalography_EEG }\end{array}$ \\
\hline 6.11 & 32.00 & 1.05 & $\begin{array}{l}\text { IS_strategy_and_business_outcomes ==> } \\
\text { Design_and_action }\end{array}$ \\
\hline 5.34 & 28.00 & 1.36 & $\begin{array}{l}\text { IS_strategy_and_business_outcomes ==> } \\
\text { Emotional_processes }\end{array}$ \\
\hline 4.58 & 24.00 & 2.42 & $\begin{array}{l}\text { IS_strategy_and_business_outcomes ==> Contribution- } \\
\text { 9. }\end{array}$ \\
\hline 3.82 & 20.00 & 1.31 & $\begin{array}{l}\text { IS_strategy_and_business_outcomes ==> Contribution- } \\
\text { 5 }\end{array}$ \\
3.05 & 16.00 & 1.61 & $\begin{array}{l}\text { IS_strategy_and_business_outcomes ==> } \\
\text { Social_processes }\end{array}$ \\
\hline
\end{tabular}

Note: S: Support, C: Confidence 


\subsection{Correspondence analysis: relationships between a pair of NeuroIS elements}

Our collected data also enable us to investigate in finer details the relationships between a pair of NeuroIS elements. In this paper, we provide an example of such pair, due to the page limitation. Among all the NeuroIS elements identified in [31], the "neuroscience tool" and "NeuroIS construct category" are two significant elements that have a significant impact on the NeuroIS research. In order to study the details of the interrelationships between these two elements, we performed a correspondence analysis [35] between this pair of elements, and generated an asymmetric correspondence analysis plot. Figure 3 shows the plot for the 20 neuroscience tools (component 1) corresponding to the 4 NeuroIS construct categories (component 2). Figure 4 shows the plot for the 3 neuroscience tool categories (component 1) corresponding to the 4 NeuroIS construct categories (component 2).

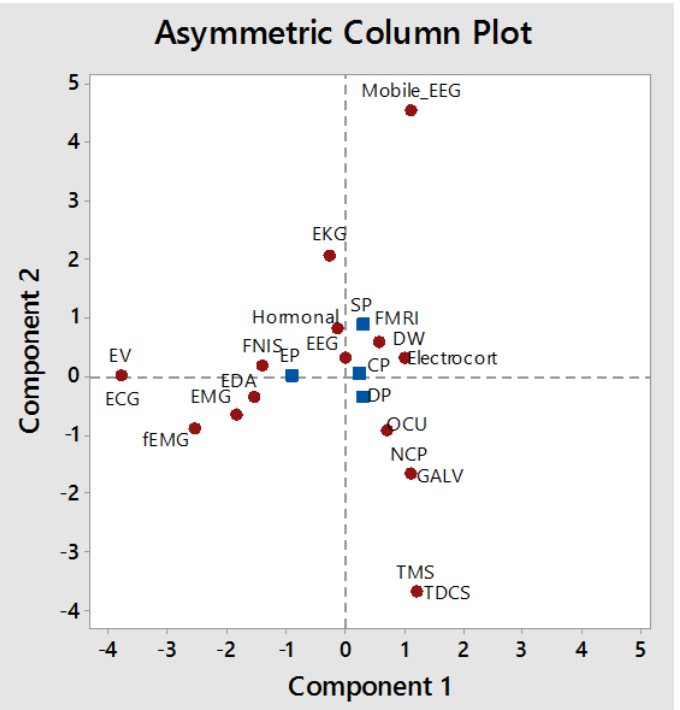

Figure 3. The correspondence analysis plot between neuroscience tool and NeuroIS construct category

In Figure 4, it is observed that among the different NeuroIS construct categories (the column component), the social process (SP) and emotional process (EP) are farther away from the origin than the cognitive process (CP) and decision making process (DP) are. This implies that SP and EP are likely to have a stronger association with the neuroscience tool (row component) than CP and DP would have. For EP, it is in close proximity with the neuroscience tool Functional Near-Infrared Spectroscopy (FNIS) and Electrodermal activity device (EDA). In either case, the angle formed between the line connecting the origin to the row component and the line connecting the origin to the column component is quite small, indicating a potential strong association between EP and FNIS, as well as between EP and EDA. In other words, the result shows that Near-Infrared Spectroscopy and Electrodermal activity devices are mainly utilized as neuroscience tools to study emotional process NeuroIS constructs.

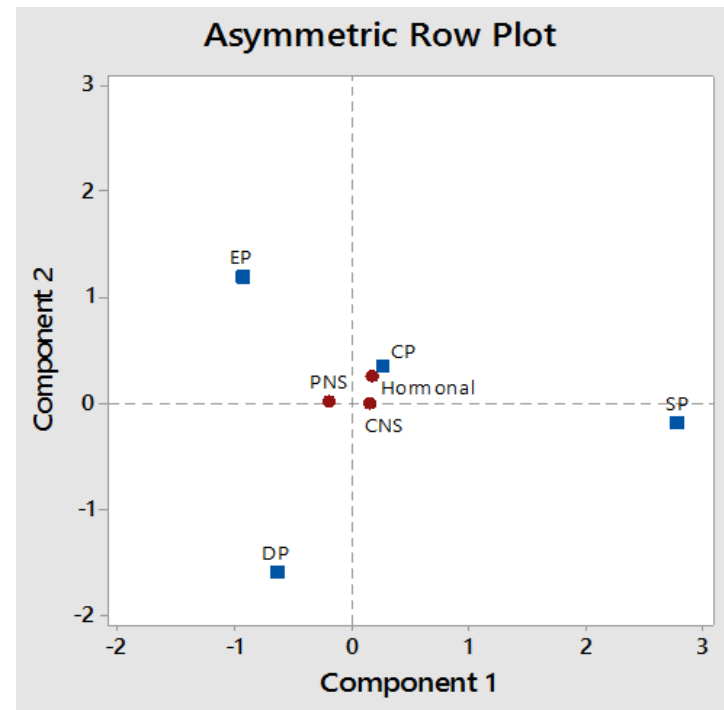

Figure 4. The correspondence analysis plot between neuroscience tool category and NeuroIS construct category

In Figure 4, it is noticed that all three neuroscience tool categories are quite close to the origin, with hormone system tools (Homonal) farthest from the origin. This may indicate that any potential association between the neuroscience tool category and NeuroIS construct category may not be very strong. Combining the results from Figure 3 and 4, we suggest that if we want to investigate the relationship between the type of NeuroIS constructs with the neuroscience tool, it is better to study the relations with the exact neuroscience tool, instead of the tool category.

\section{Conclusion, limitation and future research direction}

NeuroIS, an emerging and promising nascent field within IS, has entered its $10^{\text {th }}$ year in growing since its inception in 2007. With the increasing number of research projects focusing on NeuroIS, and the expanding appeal of NeuroIS to IS academia and industry alike, we now believe it is a perfect time to take stock and assess the state-of-the-art of NeuroIS research in its first 10 years. Therefore, we perform a systematic literature review (SLR) on the first 10 years of NeurIS research publications, following the 
PRISMA SLR framework. Based on the NeuroIS general framework described by Riedl and Léger [31], we reviewed the collected literature, identified the NeuroIS elements by devising a standard binary coding protocol for abstracting these elements from the articles, and analyzed the data to explore the relationships among those identified NeuroIS elements.

Besides the descriptive data analysis that illustrates the distribution of the literature from the perspectives of those elements, we conducted three main analyses. First, we conducted a text cluster analysis that groups our collected literature into 8 categories, indicating that the extant NeuroIS literature has eight types. We briefly discussed the properties for each of the NeuroIS research types. Then we studied the associations among various NeuroIS concepts. We conducted a market basket analysis and generated concept link diagrams to illustrate the correlations among different NeuroIS concepts. Last but not least, we conducted a correspondence analysis on a pair of identified NeuroIS elements (i.e., neuroscience tools and NeuroIS construct type) to investigate the more granulated details of the inter-relationships between these two NeuroIS elements. The results indicate that it may be better to investigate the correlation of NeuroIS construction type with specific neuroscience tools, instead of with general neuroscience tool categories.

From our SLR results, NeuroIS researchers can gain insights regarding the types of NeuroIS research, the distinct properties of those different NeuroIS research types, the interrelationship among various NeuroIS concepts, or between a specific pair of NeuroIS elements. These insights would help the NeuroIS researchers to recognize new research topics, identify significant research questions, and align their research activities with the overall patterns of NeuroIS research.

We are currently extending our analysis to other NeuroIS concepts and elements and attempting to discover more interesting insights. We are also exploring additional analytic approaches that would be appropriate to apply to the dataset, and obtain new insights to new research questions. In addition, we are making a continuous and long-term commitment to developing a repository for NeuroIS research publications. The dataset created and the analyses conducted in this paper constitute the first components of this repository. We will continue this effort in the future, and periodically and systematically review the repository to provide new insights of NeuroIS research.

\section{References}

[1] Cacioppo, J.T., G.G. Berntson, J.F. Sheridan, and M.K. McClintock, Multilevel integrative analyses of human behavior: social neuroscience and the complementing nature of social and biological approaches. Psychological bulletin, 2000. 126(6): p. 829.

[2] Wilson, E.O., Biology and the social sciences.Daedalus, 1977: p. 127-140.

[3]Cacioppo, J.T., G.G. Berntson, and H.C. Nusbaum, Neuroimaging as a new tool in the toolbox of psychological science. Current Directions in Psychological Science, 2008. 17(2): p. 62-67.

[4]Camerer, C.F., Strategizing in the brain. Science, 2003. 300(5626): p. 1673-1675.

[5]Dimoka, A., Brain mapping of psychological processes with psychometric scales: An fMRI method for social neuroscience. NeuroImage, 2011. 54: p. S263-S271.

[6]Lee, N., A.J. Broderick, and L. Chamberlain, What is 'neuromarketing'? A discussion and agenda for future research. International journal of psychophysiology, 2007. 63(2): p. 199-204.

[7]Djamasbi, S., M. Siegel, and T. Tullis. Designing noticeable bricklets by tracking users' eye movements. in System Science (HICSS), 2012 45th Hawaii International Conference on. 2012. IEEE.

[8]Adam, M.T., J. Krämer, and C. Weinhardt, Excitement up! Price down! Measuring emotions in Dutch auctions. International Journal of Electronic Commerce, 2012. 17(2): p. 7-40.

[9]Riedl, R. and A. Javor, The biology of trust: Integrating evidence from genetics, endocrinology, and functional brain imaging. Journal of Neuroscience, Psychology, and Economics, 2012. 5(2): p. 63.

[10]Pavlou, P., F. Davis, and A. Dimoka, Neuro IS: the potential of cognitive neuroscience for information systems research. ICIS 2007 Proceedings, 2007: p. 122.

[11]Riedl, R., Zum Erkenntnispotenzial der kognitiven Neurowissenschaften für die Wirtschaftsinformatik: Überlegungen anhand exemplarischer Anwendungen.

NeuroPsychoEconomics, 2009. 4(1): p. 32-44. 
[12]Dimoka, A., R.D. Banker, I. Benbasat, F.D. Davis, A.R. Dennis, D. Gefen, A. Gupta, A. Ischebeck, P. Kenning, and P.A. Pavlou, On the use of neurophysiological tools in IS research: Developing a research agenda for NeuroIS. 2010: p. 680.

[13]Riedl, R., R.D. Banker, I. Benbasat, F.D. Davis, A.R. Dennis, A. Dimoka, D. Gefen, A. Gupta, A. Ischebeck, and P. Kenning, On the foundations of NeuroIS: reflections on the Gmunden Retreat 2009. 2010.

[14]Dimoka, A., What does the brain tell us about trust and distrust? Evidence from a functional neuroimaging study. Mis Quarterly, 2010: p. 373-396.

[15]Leger, P.-M., R. Riedl, and J. vom Brocke, Emotions and ERP information sourcing: the moderating role of expertise. Industrial Management \& Data Systems, 2014. 114(3): p. 456-471.

[16]de Guinea, A.O., R. Titah, and P.-M. Léger, Explicit and implicit antecedents of users' behavioral beliefs in information systems: A neuropsychological investigation. Journal of Management Information Systems, 2014. 30(4): p. $179-210$.

[17]Riedl, R., M. Hubert, and P. Kenning, Are there neural gender differences in online trust? An fMRI study on the perceived trustworthiness of eBay offers. Mis Quarterly, 2010. 34(2): p. 397-428.

[18]Riedl, R., P.N. Mohr, P.H. Kenning, F.D. Davis, and H.R. Heekeren, Trusting humans and avatars: A brain imaging study based on evolution theory. Journal of Management Information Systems, 2014. 30(4): p. 83-114.

[19]Warkentin, M., A.C. Johnston, E. Walden, and D.W. Straub, Neural Correlates of Protection Motivation for Secure IT Behaviors: An fMRI Examination. Journal of the Association for Information Systems, 2016. 17(3): p. 194.

[20]Teubner, T., M. Adam, and R. Riordan, The impact of computerized agents on immediate emotions, overall arousal and bidding behavior in electronic auctions. Journal of the Association for Information Systems, 2015. 16(10): p. 838.

[21]Vance, A., B.B. Anderson, C.B. Kirwan, and D. Eargle, Using measures of risk perception to predict information security behavior: Insights from electroencephalography (EEG). Journal of the Association for Information Systems, 2014. 15(10): p. 679.
[22]Simon, H.A., The behavioral and social sciences. Science, 1980. 209(4452): p. 72-78. [23]Dimoka, A., How to conduct a functional magnetic resonance (fMRI) study in social science research. 2012.

[24]Riedl, R., F.D. Davis, and A.R. Hevner, Towards a NeuroIS research methodology: intensifying the discussion on methods, tools, and measurement. Journal of the Association for Information Systems, 2014. 15(10): p. I. 25]Riedl, R., H. Kindermann, A. Auinger, and A. Javor, Technostress from a neurobiological perspective. Business \& Information Systems Engineering, 2012. 4(2): p. 61-69.

[26]Tams, S., K. Hill, A.O. de Guinea, J. Thatcher, and V. Grover, NeuroIS-Alternative or complement to existing methods? Illustrating the holistic effects of neuroscience and self-reported data in the context of technostress research. Journal of the Association for Information Systems, 2014. 15(10): p. 723.

[27]Kindermann, H. and A. Javor, The Impact of Highly Activating Computer Games on Memory.

[28]Fredette, M., É. Labonté-LeMoyne, P.-M. Léger, F. Courtemanche, and S. Sénécal, Research Directions for Methodological Improvement of the Statistical Analysis of Electroencephalography Data Collected in NeuroIS, in Information Systems and Neuroscience. 2015, Springer. p. 201-206.

[29]vom Brocke, J. and T.-P. Liang, Guidelines for neuroscience studies in information systems research. Journal of Management Information Systems, 2014. 30(4): p. 211-234.

[30]Moher, D., A. Liberati, J. Tetzlaff, D.G. Altman, and P. Group, Preferred reporting items for systematic reviews and meta-analyses: the PRISMA statement. PLoS med, 2009. 6(7): p. e1000097.

[31]Riedl, R. and P.-M. Léger, Fundamentals of NeuroIS. 2016: Springer.

[32]Dimoka, A., R.D. Banker, I. Benbasat, F.D. Davis, A.R. Dennis, D. Gefen, A. Gupta, A. Ischebeck, P.H. Kenning, P.A. Pavlou, G. Müller-Putz, R. Riedl, J. vom Brocke, and B. Weber, ON THE USE OF

NEUROPHYSIOLOGICAL TOOLS IN IS RESEARCH: DEVELOPING A RESEARCH AGENDA FOR NEUROIS. MIS Quarterly, 2012. 36(3): p. 679-A19.

[33]Dimoka, A., P.A. Pavlou, and F.D. Davis, NeuroIS: The Potential of Cognitive Neuroscience for Information Systems Research. 
Information Systems Research, 2011. 22(4): p.

687-702.

[34]Can, F. and E.A. Ozkarahan, Concepts and effectiveness of the cover-coefficient-based clustering methodology for text databases. ACM Transactions on Database Systems (TODS), 1990. 15(4): p. 483-517.
[35]Hall, P., J. Dean, I.K. Kabul, and J. Silva, An overview of machine learning with $S A S \circledR$ enterprise miner ${ }^{\mathrm{TM}}$. Cary: SAS Institute Inc, 2014. 